\title{
THE EFFECT OF DIFFERENT OBSERVATIONAL DATA IN CONSTRAINING COSMOLOGICAL PARAMETERS*
}

\author{
YUNGUI GONG \\ School of Physics, Huazhong University of Science and Technology, Wuhan 430074, China \\ College of Mathematics and Physics, Chongqing University of Posts and Telecommunications, \\ Chongqing 400065, China \\ Institute of Theoretical Physics, Chinese Academy of Sciences, Beijing 100190, China \\ gongyg@cqupt.edu.cn \\ QING GAO \\ College of Mathematics and Physics, Chongqing University of Posts and Telecommunications, \\ Chongqing 400065, China \\ ZONG-HONG ZHU \\ Department of Astronomy, Beijing Normal university, Beijing 100875, China \\ zhuzh@bnu.edu.cn
}

Received 5 January 2012

\begin{abstract}
We use the SNLS3 compilation of 472 type Ia supernova data, the baryon acoustic oscillation measurement of distance, and the cosmic microwave background radiation data from the seven year Wilkinson Microwave Anisotropy Probe to study the effect of their different combinations on the fittings of cosmological parameters. Neither BAO nor WMAP7 data alone gives good constraint on the equation of state parameter of dark energy, but both WMAP7 data and BAO data help type Ia supernova data break the degeneracies among the model parameters, hence tighten the constraint on the variation of equation of state parameter $w_{a}$, and WMAP7 data does the job a little better. Although BAO and WMAP7 data provide reasonably good constraints on $\Omega_{m}$ and $\Omega_{k}$, it is not able to constrain the dynamics of dark energy, we need SNe Ia data to probe the property of dark energy, especially the variation of the equation of state parameter of dark energy. For the SNLS SNe Ia data, the nuisance parameters $\alpha$ and $\beta$ are consistent for all different combinations of the above data. Their impacts on the fittings of cosmological parameters are minimal. $\Lambda \mathrm{CDM}$ model is consistent with current observational data.
\end{abstract}

Keywords: Cosmological parameters; dark energy.

PACS numbers: 98.80.Cq, 98.80.-k

*This talk is based on the work 1110.6535 


\section{Introduction}

The accelerating expansion of the universe was first discovered in 1998 by the observations of Type Ia supernovae $(\mathrm{SNe} I \mathrm{I})^{1,2}$. As more accurate data are available, it is possible to measure the acceleration and the dynamical mechanism behind the acceleration. In the recent release of the measurements of the baryon acoustic oscillation $(\mathrm{BAO})$ peaks at redshifts $z=0.44,0.6$ and 0.73 in the galaxy correlation function of the final dataset of the WiggleZ dark energy survey ${ }^{3}$, Blake et al. used these three $\mathrm{BAO}$ data along with $\mathrm{BAO}$ data at redshifts $z=0.2$ and 0.35 measured from the distribution of galaxies ${ }^{4}$ and the measurement of BAO at redshift $z=0.106$ from the 6 -degree Field Galaxy Survey $(6 \mathrm{dFGS})^{5}$ to constrain $\Lambda \mathrm{CDM}$ model. It was found that the constraints on $\Lambda$ CDM model from BAO data are even better than those from Union2 $\mathrm{SNe}$ Ia data ${ }^{6}$. It was also found that the combination of BAO and the seven-year Wilkinson Microwave Anisotropy Probe (WMAP7) data gives much better constraints on $\Lambda \mathrm{CDM}$ model than the combination of $\mathrm{SNe}$ Ia and WMAP7 data does. Sullivan et al. found that both the three year Supernova Legacy Survey (SNLS3) SNe Ia data alone and the combination of WMAP7 and BAO data at redshifts $z=0.2$ and $z=0.35^{4}$ gave similar constraint on the equation of state parameter $w$ for the flat constant $w$ model $^{7}$. This means that the current $\mathrm{BAO}$ data is robust to constrain cosmological parameters. Because there are more than 500 SNe Ia data points, and only 6 data points in the updated BAO data ${ }^{3}$, the fitting process for BAO data is easier and faster than that for SNe Ia data. If the constraints on the property of dark energy from BAO data are tighter than those from SNe Ia data, then we just need to apply BAO data only for a faster fitting although SNe Ia data and BAO data are complementary to each other. The redshifts of BAO data span from $z=0.106$ to $z=0.73$, we may expect that BAO data catches the dynamical property of dark energy.

In this paper, we first use Chevallier-Polarski-Linder (CPL) model to test the robustness of BAO data, then study the observational constraints on the equation of state of dark energy based on different combinations of the following data: SNLS3 sample of 472 SNe Ia data with systematic errors ${ }^{8}$; the BAO measurements from the $6 \mathrm{dFGS}^{5}$, the distribution of galaxies ${ }^{4}$ and the WiggleZ dark energy survey ${ }^{3}$; and the WMAP7 data $^{9}$. In addition to studying the effects of different observational data and their combinations on the constraints of cosmological parameters, we also reconstruct the equation of state of dark energy $w(z)$ and $\operatorname{Om}(z)$ by using these data sets.

The paper is organized as follows. In section 2, we present the SNLS3 SNe Ia data $^{8}$, the BAO data ${ }^{3,4,5}$, the WMAP7 data ${ }^{9}$, and all the formulae related to these data. In section 3 , we present all the models and the fitting results, and conclusions are drawn in section 4 . 


\section{Observational data}

The SNLS3 SNe Ia data consists of 123 low-redshift SNe Ia data with $z \lesssim 0.1$ mainly from Calan/Tololo, CfAI, CfAII, CfAIII and CSP, 242 SNe Ia over the redshift range $0.08<z<1.06$ observed from the $\mathrm{SNLS}^{8}, 93$ intermediate-redshift SNe Ia data with $0.06 \lesssim z \lesssim 0.4$ observed during the first season of Sloan Digital Sky Survey (SDSS)-II supernova (SN) survey ${ }^{10}$, and 14 high-redshift SNe Ia data with $z \gtrsim 0.8$ from Hubble Space Telescope ${ }^{11}$. The SNLS3 SNe Ia data used the combination of SALT2 and SiFTO light-curve fitters ${ }^{8}$. To use the 472 SNLS3 SNe Ia data ${ }^{8}$, we minimize

$$
\chi_{s n}^{2}(\mathbf{p}, \alpha, \beta)=\sum_{i, j=1}^{472}\left(m_{B}-m_{m o d}\right)^{T} C_{s n}^{-1}\left(m_{B}-m_{m o d}\right),
$$

where $m_{B}$ is the rest-frame peak B-band magnitude of a $\mathrm{SN}$, the predicted magnitude of the SN given a cosmological model is $m_{\text {mod }}=5 \log _{10} \mathcal{D}_{L}\left(z_{\text {hel }}, z_{c m b}, \mathbf{p}\right)-$ $\alpha(s-1)+\beta \mathcal{C}+\mathcal{M}_{B}, z_{h e l}$ and $z_{c m b}$ are the heliocentric and the CMB frame redshifts of the SN, $s$ is the stretch given by the data, $\mathcal{C}$ is the color measure for the $\mathrm{SN}$ given by the data, $\alpha$ and $\beta$ are nuisance parameters used for the SNLS3 data fitting, $\mathcal{M}_{B}$ is another nuisance parameter incorporating the absolute magnitude and Hubble constant and it is marginalized over in the SN fitting process because of the arbitrary normalization of the magnitude, $C_{s n}\left(z_{i}, z_{j}\right)$ is the covariant matrix which includes both the systematical and statistical uncertainties for the SNe Ia data ${ }^{8}$. The correction on the dependence of the host-galaxy stellar mass is also included. The Hubble-constant free luminosity distance $\mathcal{D}_{L}(z)$ is

$$
\mathcal{D}_{L}(z)=H_{0} d_{L}(z)=\frac{1+z}{\sqrt{\left|\Omega_{k}\right|}} S_{k}\left[\sqrt{\left|\Omega_{k}\right|} \int_{0}^{z} \frac{d x}{E(x)}\right],
$$

where the dimensionless Hubble parameter $E(z)=H(z) / H_{0}$, and $S_{k}(x)$ is defined as $x, \sin (x)$ or $\sinh (x)$ for $k=0,+1$, or -1 , respectively. For the fitting to the SNL3 data, we need to add two more nuisance parameters $\alpha$ and $\beta$ in addition to the model parameters $\mathbf{p}$ and the nuisance parameter $\mathcal{M}_{B}$.

For the BAO data, we use the measurements from the $6 \mathrm{dFGS}^{5}$, the distribution of galaxies ${ }^{4}$ and the WiggleZ dark energy survey ${ }^{3}$. Percival et al. measured the distance ratio ${ }^{4}$,

$$
d_{z}=\frac{r_{s}\left(z_{d}\right)}{D_{V}(z)}
$$

at two redshifts $z=0.2$ and $z=0.35$ to be $d_{0.2}^{o b s}=0.1905 \pm 0.0061$, and $d_{0.35}^{\text {obs }}=$ $0.1097 \pm 0.0036$. Here the effective distance is

$$
D_{V}(z)=\left[\frac{d_{L}^{2}(z)}{(1+z)^{2}} \frac{z}{H(z)}\right]^{1 / 3}
$$

$z_{d}$ is the drag redshift defined in Ref. 12 , the comoving sound horizon is

$$
r_{s}(z)=\int_{z}^{\infty} \frac{c_{s}(x) d x}{E(x)}
$$


where the sound speed $\left.c_{s}(z)=1 / \sqrt{3\left[1+\bar{R}_{b} /(1+z)\right.}\right]$, and $\bar{R}_{b}=3 \Omega_{b} h^{2} /(4 \times 2.469 \times$ $\left.10^{-5}\right)$. Beutler et al. derived that $d_{0.106}^{\text {obs }}=0.336 \pm 0.015^{5}$. The WiggleZ dark energy survey measured the acoustic parameter

$$
A(z)=\frac{D_{V}(z) \sqrt{\Omega_{m} H_{0}^{2}}}{z},
$$

at three redshifts $z=0.44, z=0.6$ and $z=0.73$, and the results and their covariance matrix are listed in table 3 and table 2 in Ref. 3 . To use the BAO data, we minimize

$$
\begin{aligned}
\chi_{B a o}^{2}\left(\mathbf{p}, \Omega_{b} h^{2}, h\right)= & \sum_{i, j=1}^{2} \Delta d_{i} C_{d z}^{-1}\left(d_{i}, d_{j}\right) \Delta d_{j}+\frac{\left(d_{0.106}-0.336\right)^{2}}{0.015^{2}} \\
& +\sum_{i, j=1}^{3} \Delta A_{i} C_{A}^{-1}\left(A_{i}, A_{j}\right) \Delta A_{j},
\end{aligned}
$$

where $d_{i}=\left(d_{z=0.2}, d_{z=0.35}\right), \Delta d_{i}=d_{i}-d_{i}^{\text {obs }}$ and the covariance matrix $C_{d z}\left(d_{i}, d_{j}\right)$ for $d_{z}$ at $z=(0.2,0.35)$ is taken from equation (5) in Ref. $4 ; A_{i}=$ $(A(0.44), A(0.6), A(0.73)), \Delta A_{i}=A\left(z_{i}\right)-A\left(z_{i}\right)^{\text {obs }}$ and the covariance matrix $C_{A}\left(A_{i}, A_{j}\right)$ for the data points $A(z)$ at $z=(0.44,0.6,0.73)$ is taken from table 2 in Ref. 3. Besides the model parameters $\mathbf{p}$, we need to add two more nuisance parameters $\Omega_{b} h^{2}$ and $\Omega_{m} h^{2}$ when we use the BAO data.

For the WMAP7 data, we use the measurements of the three derived quantities: the shift parameter $R\left(z^{*}\right)$ and the acoustic index $l_{A}\left(z^{*}\right)$ at the recombination redshift $z^{*}$. In particular, we minimize

$$
\chi_{C M B}^{2}\left(\mathbf{p}, \Omega_{b} h^{2}, h\right)=\sum_{i, j=1}^{3} \Delta x_{i} C_{C M B}^{-1}\left(x_{i}, x_{j}\right) \Delta x_{j},
$$

where the three parameters $x_{i}=\left[R\left(z^{*}\right), l_{A}\left(z^{*}\right), z^{*}\right], \Delta x_{i}=x_{i}-x_{i}^{o b s}$ and the covariance matrix $C_{C M B}\left(x_{i}, x_{j}\right)$ for the three parameters is taken from Table 10 in Ref. 9. The shift parameter $R$ is expressed as

$$
R\left(z^{*}\right)=\frac{\sqrt{\Omega_{m}} \mathcal{D}_{L}\left(z^{*}\right)}{1+z^{*}}=1.725 \pm 0.018 .
$$

The acoustic index $l_{A}$ is

$$
l_{A}\left(z^{*}\right)=\frac{\pi d_{L}\left(z^{*}\right)}{\left(1+z^{*}\right) r_{s}\left(z^{*}\right)}=302.09 \pm 0.76
$$

and $z^{*}=1090.04 \pm 0.93$ is the redshift at the recombination whose parametrization was defined in Ref. 13. We also need to add the nuisance parameters $\Omega_{b} h^{2}$ and $\Omega_{m} h^{2}$ to the parameter space when we employ the WMAP7 data.

To determine the model parameters $\mathbf{p}$, we minimize

$$
\chi^{2}=\chi_{s n}^{2}+\chi_{B a o}^{2}+\chi_{C M B}^{2}
$$


After fitting the observational data to different dark energy models by using the Monte Carlo Markov Chain method, we apply the $O m$ diagnostic $^{14}$ to detect the deviation from the $\Lambda$ CDM model. For a flat universe,

$$
O m(z)=\frac{E^{2}(z)-1}{(1+z)^{3}-1} .
$$

For the flat $\Lambda$ CDM model, $\operatorname{Om}(z)=\Omega_{m}$ is a constant which is independent of the value of $\Omega_{m}$. Because of this property, Om diagnostic is less sensitive to observational errors than the equation of state parameter $w(z)$ does. On the other hand, the bigger the value of $\operatorname{Om}(z)$, the bigger the value of $w(z)$, so the behavior of $\operatorname{Om}(z)$ catches the dynamical property of $w(z)$.

We also apply the figure of merit (FOM) proposed by the Dark Energy Task Force ${ }^{15}$ as a diagnostic tool to compare the effectiveness of different combinations of observational data on constraining the equation of state parameters $w_{0}$ and $w_{a}$ in CPL model. FOM is defined as the the reciprocal of the area of the error ellipse enclosing the $95 \%$ confidence limit in the $w_{0}-w_{a}$ plane, it is proportional to $\left[\operatorname{det} C_{w}\left(w_{0}, w_{a}\right)\right]^{-1 / 2}$, here $C_{w}\left(w_{0}, w_{a}\right)$ is the correlation matrix of $w_{0}$ and $w_{a}$.

\section{Cosmological fitting results}

\section{1. $\Lambda$ CDM model}

We first review the effects of different observational data on $\Lambda$ CDM model. In Fig. 11 of Ref. 6, Amanullah et al. compared the constraint on $\Lambda$ CDM model from Union2 SNe Ia data with that from BAO data, and they found that $\Omega_{\Lambda}$ is not sensitive to the BAO data. In figure 15 of Ref. 3, Blake et al. compared the constraints on $\Omega_{m}$ and $\Omega_{k}$ from the updated BAO data ${ }^{3}$ under the assumption that $\Omega_{b} h^{2}=0.02227$ with those from Union2 SNe Ia data, and they found that the constraints are similar, and the constraint on $\Omega_{m}$ is much better by using BAO data than that by using $\mathrm{SNe}$ Ia data. Moreover, Blake et al. found that the constraint from the combination of Union2 SNe Ia, BAO and WMAP7 data is similar to that from the combination of BAO and WMAP7 data ${ }^{3}$.

For comparison, we show the constraints on $\Lambda \mathrm{CDM}$ from different data sets in Figure 1. Note that we set $\Omega_{b} h^{2}$ as a free nuisance parameter in applying updated BAO data. In Figure 1, we also show the constraints on $\Lambda \mathrm{CDM}$ model from the previous BAO distance ratios at redshifts $z=0.2$ and $z=0.35^{4}$ (the cyan lines). For simplicity, we also assume that $\Omega_{b} h^{2}=0.02227$ and $h=0.738$ when we apply the previous BAO data.

These results along with the constraints on the nuisance parameters $\alpha$ and $\beta$ are summarized in Table 1 . The error bars of $\alpha$ and $\beta$ are around 0.1 , and they are consistent at $1 \sigma$ level for different fittings. The best fit value of $\Omega_{m}$ from SNe Ia data is marginally consistent with that from BAO data, this shows a little tension between SN Ia and BAO data. 

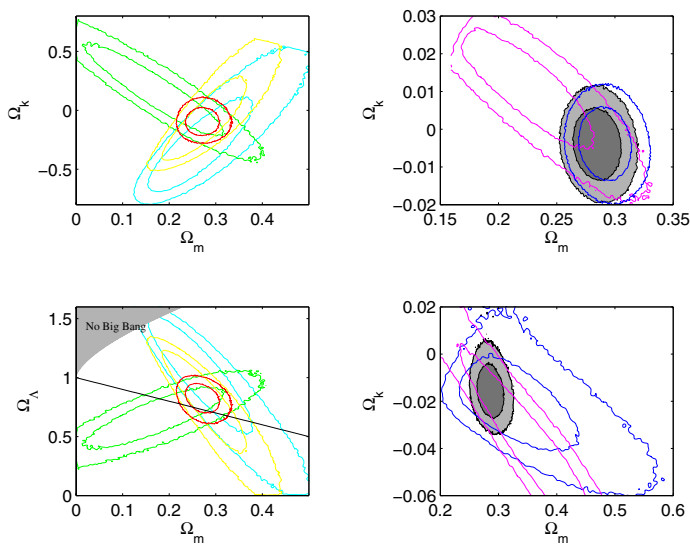

Fig. 1. The marginalized $1 \sigma$ and $2 \sigma$ contour plots. The upper panels show the constraints on $\Omega_{m}$ and $\Omega_{k}$ for the $\Lambda \mathrm{CDM}$ model. The lower left panel shows the constraints on $\Omega_{m}$ and $\Omega_{\Lambda}$ for the $\Lambda$ CDM model, the solid black line represents the flat case. The lower right panel shows the constraints on $\Omega_{m}$ and $\Omega_{k}$ for the CPL model. The green lines label the constraints from $\mathrm{SNe}$ Ia data only, the yellow lines label the constraints from BAO data only, the cyan lines label the constraints from BAO distance ratios measured by Percival et al. and it is assumed that $\Omega_{b} h^{2}=0.02227$ and $h=0.738$, the red lines label the constraints from the combination of SNe Ia and $\mathrm{BAO}$ data, the magenta lines label the constraints from the combination of SNe Ia and WMAP7 data, the blue lines label the constraints from the combination of WMAP7 and BAO data, the shaded regions label the constraints from the combination of SNe Ia, BAO and WMAP7 data.

Table 1. The marginalized $1 \sigma$ errors for $\Omega_{m}$ and $\Omega_{k}$ in $\Lambda$ CDM model constrained by different observational data

\begin{tabular}{ccccc}
\hline Data & $\Omega_{m}$ & $\Omega_{k}$ & $\alpha$ & $\beta$ \\
\hline SNe Ia & $0.17_{-0.09}^{+0.1}$ & $0.15 \pm 0.25$ & $1.423_{-0.094}^{+0.122}$ & $3.267_{-0.109}^{+0.112}$ \\
\hline BAO & $0.26_{-0.03}^{+0.09}$ & $-0.16_{-0.11}^{+0.38}$ & & \\
\hline SNe+BAO & $0.27 \pm 0.02$ & $-0.11_{-0.07}^{+0.09}$ & $1.428_{-0.102}^{+0.114}$ & $3.249_{-0.095}^{+0.125}$ \\
\hline SNe+WMAP7 & $0.22_{-0.03}^{+0.05}$ & $0.01 \pm 0.01$ & $1.422_{-0.095}^{+0.121}$ & $3.26_{-0.106}^{+0.115}$ \\
\hline BAO+WMAP7 & $0.29_{-0.01}^{+0.02}$ & $-0.004_{-0.006}^{+0.007}$ & & \\
\hline SNe+BAO+WMAP7 & $0.28 \pm 0.01$ & $-0.004_{-0.007}^{+0.006}$ & $1.41_{-0.088}^{+0.127}$ & $3.261_{-0.116}^{+0.104}$ \\
\hline
\end{tabular}

It is clear that the updated BAO data ${ }^{3}$ greatly improves the constraint over the previous BAO data ${ }^{4}$, and the ability of constraining $\Lambda$ CDM by BAO data is even better than that by SNe Ia data. In the next section, we compare the constraints on dynamical property of dark energy from different data sets.

\section{2. $C P L$ parametrization}

In this section, we apply the CPL parametrization ${ }^{16,17}$,

$$
w(z)=w_{0}+\frac{w_{a} z}{1+z},
$$


to test the effects of different combinations of data in constraining the property of dark energy. In this model, we have four model parameters $\mathbf{p}=\left(\Omega_{m}, \Omega_{k}, w_{0}, w_{a}\right)$. From the results of $\Lambda \mathrm{CDM}$ model, we know the constraints on $\Omega_{k}$ from either SNe Ia data alone or BAO data alone are not good. For the curved CPL model, due to the addition of two more model parameters, we expect the situation becomes worse and in fact this is true. So we only consider the combinations of SNLS3 SNe Ia and/or BAO data with WMAP7 data. The contours are shown in Figs. 1 and 2. The $1 \sigma$ results along with the constraints on the nuisance parameters $\alpha$ and $\beta$ are summarized in Table 2. As expected, we see that the constraint on $\Omega_{k}$ from the combination of BAO and WAMP7 data (the blues lines) is better than that from the combination of SNe Ia and WMAP7 data (the magenta lines). The $\Omega_{m^{-}}$ $\Omega_{k}$ contour becomes much smaller when we combine SNe Ia, BAO and WMAP7 data (the shaded regions). From the $w_{0}-w_{a}$ contours in Figure 2, we see that the constraints from the combination of SNe Ia and WMAP7 data are much better than those from the combination of BAO and WMAP7 data, and the FOM is almost 5 times larger. The uncertainties in $w_{a}$ from the combination of SNe Ia, BAO and WMAP7 data are reduced more than half compared with those from the combination of SNe Ia and WMAP7 data, the FOM increase more than 4 times. When SNe Ia data is combined with other data, $\Omega_{k}$ is degenerated with $w_{0}$ and $w_{a}$. As $\Omega_{k}$ increases, the uncertainties in $w_{a}$ reduced. However, for the combination of BAO and WMAP7 data, the degeneracies between $\Omega_{k}$ and $w_{0}$ and $w_{a}$ become weaker. Therefore, although SNe Ia data does not provide tight constraints on $\Omega_{m}$ and $\Omega_{k}$, its constraint on the equation of state parameter of dark energy is much better. $\Lambda$ CDM model (the cross) is outside the $1 \sigma$ contour when we use the combination of SNe Ia, BAO and WMAP7 data (the shaded regions). By using the constraints from the combination of all observational data, we reconstruct $w(z)$ and the result is shown in Fig. 2.

Since WMAP7 data greatly reduces the error on $\Omega_{k}$, its effect on flat CPL model still needs to be studied. In Figure 2, we show the marginalized $w_{0}-w_{a}$ contour plots constrained from different combinations of data. The $1 \sigma$ results along with the constraints on the nuisance parameters $\alpha$ and $\beta$ are summarized in Table 2. The constraints on $w_{0}$ and $w_{a}$ from BAO data alone are not good, so the results are not shown. The constraint from SNe Ia data alone is similar to that from the combination of SNe Ia and WMAP7 data in the curved case. This is easily understood because the addition of WMAP7 data in the curved case is used to limit $\Omega_{k}$ around zero. When the BAO data is added to the SNe Ia data, the uncertainty in $w_{a}$ is reduced more than half and the FOM becomes 5 times larger. When WMAP7 data is added to the SNe Ia data, the uncertainty in $w_{a}$ is reduced a little further and the FOM becomes almost 10 times larger. Compared with the constraint from the combination of BAO and WMAP7 data, we see that the SNe Ia data constrains better on $w_{0}$ and the FOM is just one half smaller, both BAO and WMAP7 data help reduce the uncertainties in $w_{a}$, the help from WMAP7 data is a little better. When 

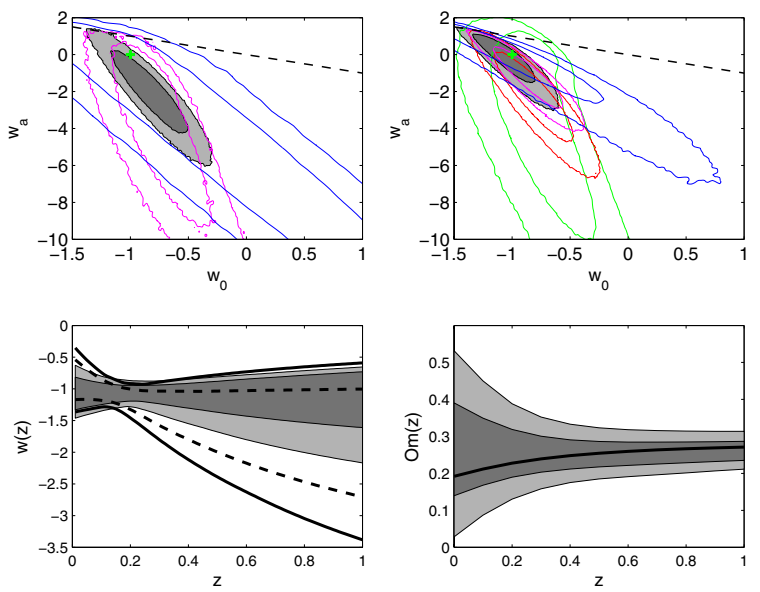

Fig. 2. The marginalized $1 \sigma$ and $2 \sigma$ constraints on the CPL model from observations. The $w_{0}$ and $w_{a}$ contours for the curved and flat CPL models are shown in the upper left and right panels, respectively. The meaning of different colors is the same as that in Figure 1. The dashed line in the $w_{0}-w_{a}$ contour denotes the condition $w_{0}+w_{a}=0$. The + sign denotes the point corresponding to the $\Lambda \mathrm{CDM}$ model. In the lower left panel, we reconstruct the evolution of $w(z)$ by using the constraints from the combination of SNe Ia, BAO and WMAP7 data, the shaded regions are for flat CPL model, and the black lines are for curved CPL model. In the lower right panel, we reconstruct $O m(z)$ by using the constraints from the combination of all data for flat CPL model, the solid line is obtained by using the best fitting values of $\Omega_{m}, w_{0}$ and $w_{a}$.

Table 2. The marginalized $1 \sigma$ constraints on CPL model by different observational data

\begin{tabular}{|c|c|c|c|c|c|c|c|}
\hline Data & $\Omega_{m}$ & $\Omega_{k}$ & $\overline{w_{0}}$ & $\overline{w_{a}}$ & $\bar{\alpha}$ & $\bar{\beta}$ & FoM \\
\hline $\begin{array}{c}\text { BAO+ } \\
\text { WMAP7 }\end{array}$ & $0.35 \pm 0.07$ & $-0.023 \pm 0.013$ & $0.4 \pm 1.4$ & $-8.6_{-7.2}^{+7.4}$ & & & 0.36 \\
\hline $\begin{array}{c}\text { SNe+ } \\
\text { WMAP7 }\end{array}$ & $0.33_{-0.06}^{+0.09}$ & $-0.03_{-0.03}^{+0.02}$ & $-0.8_{-0.2}^{+0.3}$ & $-3.4_{-5.0}^{+2.1}$ & $1.430_{-0.106}^{+0.111}$ & $3.262_{-0.104}^{+0.117}$ & 1.67 \\
\hline $\begin{array}{c}\mathrm{SNe}+\mathrm{BAO}+ \\
\text { WMAP7 }\end{array}$ & $0.28_{-0.01}^{+0.02}$ & $-0.015_{-0.008}^{+0.007}$ & $-0.8 \pm 0.2$ & $-2.02_{-1.66}^{+1.35}$ & $1.410_{-0.082}^{+0.133}$ & $3.245_{-0.084}^{+0.137}$ & 7.29 \\
\hline $\mathrm{SNe}$ & $0.31_{-0.07}^{+0.09}$ & & $-0.8_{-0.2}^{+0.4}$ & $-3.1_{-5.9}^{+2.4}$ & $1.432_{-0.107}^{+0.109}$ & $3.255_{-0.099}^{+0.123}$ & 1.15 \\
\hline $\mathrm{SNe}+\mathrm{BAO}$ & $0.28_{-0.02}^{+0.03}$ & & $-0.83_{-0.19}^{+0.26}$ & $-2.11_{-1.95}^{+1.27}$ & $1.418_{-0.090}^{+0.126}$ & $3.275_{-0.112}^{+0.110}$ & 5.94 \\
\hline $\begin{array}{c}\text { SNe+ } \\
\text { WMAP7 }\end{array}$ & $0.24_{-0.02}^{+0.03}$ & & $-0.9 \pm 0.2$ & $-1.1_{-1.4}^{+0.8}$ & $1.413_{-0.083}^{+0.133}$ & $3.284_{-0.122}^{+0.1}$ & 10.39 \\
\hline $\begin{array}{c}\text { BAO+ } \\
\text { WMAP7 }\end{array}$ & $0.30 \pm 0.04$ & & $-0.81_{-0.59}^{+0.58}$ & $-0.90_{-1.91}^{+1.96}$ & & & 2.38 \\
\hline $\begin{array}{c}\mathrm{SNe}+\mathrm{BAO}+ \\
\text { WMAP7 }\end{array}$ & $0.28_{-0.01}^{+0.02}$ & & $-1.12_{-0.07}^{+0.27}$ & $0.32_{-1.63}^{+0.21}$ & $1.429_{-0.103}^{+0.112}$ & $3.261_{-0.109}^{+0.111}$ & 12.52 \\
\hline
\end{tabular}

the combined SNe Ia, BAO and WMAP7 data are used, we get better constraints on $w_{0}$ and $w_{a}$. By using the constraints from the combination of all observational data, we reconstruct $w(z)$ and $O m(z)$ and the results are shown in Fig. 2. $\Lambda$ CDM model is consistent with almost all the combinations of different data at $1 \sigma$ level. 


\section{Conclusions}

Comparing the figure 11 of Ref. 6 with the figure 15 of Ref. 3 or Fig. 1 above, we find that the updated BAO data greatly improves the constraints on $\Lambda$ CDM model, and the constraint on $\Omega_{m}$ is much better by using BAO data than that by using SNe Ia data. Therefore we may wonder if we can use BAO data alone to constrain the property of dark energy although it was well known that BAO data alone or WMAP data alone does not provide good constraints on dark energy. Applying $\mathrm{BAO}$ data to $\mathrm{CPL}$ model, we find that the constraints on the dynamical behavior of dark energy from BAO data alone are much worse than those from SNe Ia data alone. Although SNe Ia data alone is not able to provide good constraints on $\Omega_{m}$ and $\Omega_{k}$, it provides much better constraint on the equation of state parameter of dark energy compared with that from BAO and WMAP7 data alone.

WMAP7 data helps reduce the uncertainties in $\Omega_{k}$ and BAO data helps reduce the uncertainties in $\Omega_{m}$. Neither BAO nor WMAP7 data alone gives good constraint on $w_{0}$ and $w_{a}$, but both WMAP7 data and BAO data help SNe Ia data break the degeneracies among the model parameters, hence tighten the constraint on the variation of equation of state parameter $w_{a}$, and WMAP7 data does the job a little better. The constraints on the dynamical behavior of dark energy from BAO data alone are much worse than those from SNe Ia data alone. Although BAO and WMAP7 data provide reasonably good constraints on $\Omega_{m}$ and $\Omega_{k}$, it is not able to constrain the dynamics of dark energy. SNLS3 SNe Ia data alone does not provide good constraints on $\Omega_{m}$ and $\Omega_{k}$, but it provides good constraints on the parameters $w_{0}$ and $w_{a}$ compared with those from BAO and WMAP7 data alone, so it is necessary to apply SNe Ia data to probe the dynamical property of dark energy. Due to the degeneracies among the model parameters, we need to measure $\Omega_{m}$ and $\Omega_{k}$ more precisely in order to better probe the property of dark energy. In other words, since the way that the model parameters are degenerated is different for SNe Ia, BAO and WMAP7 data, we need to combine different observational data as long as the tensions among those data are not too big. The SNLS3 SNe Ia data fitting parameters $\alpha$ and $\beta$ are consistent at $1 \sigma$ level for different combinations of SNe Ia with other data. Their impacts on the fitting of cosmological parameters are minimal.

\section{Acknowledgments}

This work was partially supported by the National Basic Science Program (Project 973) of China under grant Nos. 2007CB815401 and 2010CB833004, the NNSF of China under grant Nos. 10935013 and 11175270, the Project of Knowledge Innovation Program (PKIP) of Chinese Academy of Sciences, Grant No. KJCX2.YW.W10, CQ CSTC under grant No. 2009BA4050 and CQ CMEC under grant No. KJTD201016. Z-HZ was partially supported by the NNSF Distinguished Young Scholar project under Grant No. 10825313. 


\section{References}

1. A. G. Riess and et al., Astron. J. 116, 1009 (1998).

2. S. Perlmutter et al., Astrophys. J. 517, 565 (1999).

3. C. Blake et al., arXiv: 1108.2635 (2011).

4. W. J. Percival et al., Mon. Not. R. Astron. Soc. 401, 2148 (2010).

5. F. Beutler et al., arXiv: 1106.3366 (2010).

6. R. Amanullahet et al., Astrophys. J. 716, 712 (2010).

7. M. Sullivan et al., arXiv: 1104.1444 (2011).

8. A. Conley et al., arXiv: 1104.1443 (2011).

9. E. Komatsu et al., Astrophys. J. Suppl. 192, 18 (2011).

10. R. Kessler et al., Astrophys. J. Suppl. 185, 32 (2010).

11. A. G. Riess et al., Astrophys. J. 695, 98 (2007).

12. D. J. Eisenstein and W. Hu, Astrophys. J. 496, 605 (1998).

13. W. Hu and N. Sugiyama, Astrophys. J. 471, 542 (1996).

14. V. Sahni, A. Shafieloo and A. A. Starobinsky, Phys. Rev. D 78, 103502 (2008).

15. A. Albrecht et al., arXiv: astro-ph/0609591 (2006).

16. M. Chevallier and D. Polarski, Int. J. Mod. Phys. D 10, 213 (2001).

17. E. V. Linder, Phys. Rev. Lett. 90, 091301 (2003). 